\title{
The electric boat charging problem
}

\author{
Daniel Villa ${ }^{\mathrm{a}}$ (D), Alejandro Montoya ${ }^{\mathrm{a*}}$ (D), Juan M. Ciro ${ }^{\mathrm{b}}$ \\ aDepartamento de Ingeniería de Producción, Universidad EAFIT, Medellín, Colombia

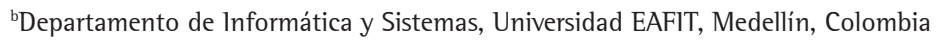 \\ *jmonto36@eafit.edu.co
}

\begin{abstract}
Paper aims: This paper studies a new optimization problem called the Electric Boat Charging Problem (EBCP), which is based on the application of electric mobility in a river transport operation problem.

Originality: This work pioneers the studies of the electric mobility on the river operations, by proposing the EBCP. This problem includes real features of the electric mobility such as nonlinear charging functions, battery degradation costs, and speed variation.

Research method: For solving the EBCP, we propose a Mixed-Integer Linear Programming (MILP) formulation. For testing our MILP formulation, we use a set of instances based on a future transport operation. We also analyze the impact of some problem parameters on the objective function, and decision variables.

Main findings: Our MILP formulation is capable to optimally solve different type of instances in competitive CPU times. The battery capacity and a time limit constraint have and important impact on the objective function and the decision-making variables.

Implications for theory and practice: We model the EBCP as a MILP formulation. This model allows to optimally solve industrial scale instances. Moreover, using a sensitivity analysis, we unveil that both the battery capacity and the time limit constraint of the EB route are critical parameters.
\end{abstract}

Keywords

Electric boat. Charging decisions. Battery degradation. MILP formulation.

How to cite this article: Villa, D., Montoya, A., \& Ciro, J. M. (2019). The electric boat charging problem. Production, 29, e20190067. https://doi.org/10.1590/0103-6513.20190067.

Received: June 29, 2019; Accepted: Aug. 12, 2019.

\section{Introduction}

Nowadays, air pollution and greenhouse gas emissions are a major concern given their impact on human health and global warming. The transport sector represents one of the major sources of pollution. In the Latin American context, the transport sector accounted for 34.1\% of the total $\mathrm{CO}_{2}$ emissions in 2005 (Timilsina \& Shrestha, 2009).

Electric Vehicles (EVs) are an alternative to replace Internal Combustion Vehicles (ICVs). Consequently, recent developments focus on road transport; however, it is also important to study the river transport operations with Electric Boats (EBs). The river transport operation is an important mean of transportation that allows connecting isolated areas, and it is the cheapest way to move passengers and freight. In some countries, the river transport operations are significant for moving goods. For example, in Colombia about 3.4 million tons of freight and 3.7 millions of passengers were mobilized by means of river transport (Arcadis Nederland BV, 2015). Considering the importance of river transport, EBs can be seen as clean alternatives to Internal Combustion Boats (ICBs), attempting to reduce the use of fossil fuels and noise. Decreasing the latter using EBs can reduce the negative impacts on the river fauna (Habib et al., 2007; Mitson, 1995). 
Nowadays, there are different river transport operations that are using EBs. Jaimurzina et al. (2017) studied the insertion of electric mobility alternatives for river transport in Latin America. They present a technological solution including considerations of generation and autonomy. They tested an EB in the Putumayo River, in Ecuador. Alvarado Ponce (2017) presented a study considering electro solar boats for Napo River operation, in Ecuador. They also presented other electro mobility solutions: one in the Tapiche River, in Peru with seven electric propelled boats; and another one for a bay operation, the SOLARIS project, in the Galapagos lslands, Ecuador.

Although EBs have shown several advantages, they also present some technical limitations such as autonomy, long charging time, and battery degradation. These shortcomings are also presented in the EVs. The lack of autonomy is caused by the limited capacity of the batteries, which leads to the necessity of visiting intermediate CSs or managing the energy consumption of the EBs. Even though the energy consumption depends on fixed features such as weight and shape of the EB, there are other factors the driver can manage that influence the consumption. One of these factors is the speed, which has a cubic impact on the consumption (Minami \& Yamachika, 2004). Another technical limitation of the EBs is the charging time. In the current best conditions, using fast chargers, the charging time is almost 20 minutes to fully charge a battery of $20 \mathrm{kWh}$ of capacity (Sadeghi-Barzani et al., 2014). Considering longer charging times, charging decisions become essential given their impact on travel itineraries (Pelletier et al., 2016). Finally, battery degradation is a technical limitation that occurs due to both calendar and cyclic behavior. Calendar aging refers to storage conditions and pass of time. Cyclic aging refers to the discharging and charging conditions to which the battery is subjected during its lifecycle (Barré et al., 2013; Pelletier et al., 2017).

Considering the technical limitations of EBs, it is necessary for any transport operation with EBs to make smart decisions regarding both EB speed and where/how much to charge. Particularly, on this paper, we focus on a common river transport operation. In this operation, the EB departs from an origin, and continues through a fixed route (the river) until its final destination. In this case, for the operation with an EB, we consider that a set of CSs have already been installed along the route. Each CS has a selection of power levels to choose from, which affect the charging time. For determining the charging time of an EB, we consider the nonlinear charging functions, which relates the battery level and the charging time (Montoya et al., 2017). We also consider that the energy price at each CS is different, and it varies for the power levels within the CS. In this study, we consider that the river is divided in a set of segments. There are two criteria for ending a segment, when there is a significant change in the river speed or when there is a CS. We assume that the EB must reach its destination before a given time limit. For the smart decision making on EB speed and where/how much to charge, we solve an optimization problem that we call the Electric Boat Charging Problem (EBCP). In this problem, the objective function is to minimize the sum of the costs of the charged energy plus battery degradation costs, taking into account autonomy and the time limit constraints. For solving this optimization problem, we propose a Mixed-Integer Linear Programming (MILP) model. This MILP formulation is able to optimally solve a set of instances inspired by a real river transport operation, in reasonable CPU times.

The remainder of this paper is organized as follows. Section 2 makes a review of the literature related to this problem. Section 3 presents some key components for the formulation of the model. Section 4 formally introduce the EBCP and presents its MILP formulation. Section 5 presents the computational experiments. Finally, section 6 concludes the paper.

\section{Literature review}

Considering that the EBCP involves both EB speed decision on each segment of the route, and the charging decisions, we divide the literature review in three parts. The first part discusses the literature related with the charging decisions, followed by a discussion on the literature concerning about the speed decision on the ICVs and ICBs, and finally we talk about a few literature related to speed decision on the EVs.

Considering the importance of the electric mobility during the last years, several authors have studied different transportation problems that include charging decisions. However, to the best of our knowledge, there are no studies focused on EBs. Nonetheless, the charging decision of an EB follows the same idea of the EVs. In the literature, there are significant number of studies about transportation problems with EVs. For instances, Pelletier et al. (2016) made a review of Vehicle Routing Problems (VRP) with EVs, showing how charging decisions are integrated with routing decisions. In the context of personal usage of EVs, Yi \& Shirk (2018) presented a charging optimization strategy using a programming algorithm.

The speed decision on the ICVs has been widely researched on transportation planning problems. For instance, Bektaş \& Laporte (2011) presented the Pollution-Routing Problem (PRP), which is an extension of the well-known VRP. The objective on this problem is to reduce the total cost of emissions. They quantified the emissions by 
considering fuel consumption as a function of the speed and weight of the freight of the ICV. Speed is then a decision variable that directly affects the solutions. From the perspective of maritime transport, liner ships operate in a fixed route context where the ports to visit and the visiting order are previously defined. Fagerholt et al. (2010) presented a nonlinear optimization strategy for speed determination on a ship operation, in order to reduce fuel emissions considering time windows. Norstad et al. (2011) presented a ship routing model for a tramp shipping operation where they consider fixed routes for speed optimization. Psaraftis \& Kontovas (2014) presented a review on speed optimization problems for maritime transportation.

Regarding the speed decision on EVs, Baum et al. (2014) studied a shortest path problem with an EV. This problem seeks to minimize both travel time and energy consumption considering speed decisions. This is based on that increasing speed will reduce travel time but affecting consumption. Betancur et al. (2017) tackled a fixed route transport problem with a Solar Electric Vehicle (SEV). In that problem, the authors had to decide the SEV speed for a set of segments on the fixed-route, and the energy comes from a solar panel installed on the SEV. However, none of these studies has included charging decisions.

Although speed optimization has been well addressed from various perspectives in both road and maritime transport, as far as we know, there are no optimization problems addressed to river transport operation planning with EBs that consider speed and charging decisions seeking to minimize both charging and battery degradation costs.

\section{EBCP components}

Before formally defining the EBCP, we briefly explain some important components related with the electric mobility incorporated in the EBCP.

\subsection{Energy consumption estimation}

Considering the autonomy limitation, the energy consumption estimation is a crucial data for the transportation planning problems for EVs and EBs. For that reason, several authors have proposed different approaches for estimating the energy consumption of the EVs. Villa \& Montoya (2018) have reviewed and classified the literature of the approaches for estimating the energy consumption of the EVs. In terms of the EBs, Minami \& Yamachika (2004) reviewed different models of the literature and proposed one model for estimating the energy consumption. The authors tested different models and concluded that the power is almost proportional to the cube of the speed. It means that the speed has an important impact over the energy consumption. For this reason, it is important to include the EB speed as a decision variable. For the EBCP, the energy consumption is an input parameter expressed in terms of the segment of the river and the EB speed. This parameter is estimated using the models presented by Minami \& Yamachika (2004).

\subsection{Nonlinear charging function}

The charging function models the relationship between battery charging time and battery level. In general, the charging function is nonlinear, because the terminal voltage and current change during the charging process. The charging scheme is composed of two phases: constant current (CC) and constant voltage (CV). In CC the current is constant until the battery's terminal voltage reaches a maximum value. During this phase, the battery level increases linearly. In the CV phase the terminal voltage is held constant at its maximum value, causing that the current decrease in an exponential way, thus generating a concave behavior (Pelletier et al., 2017). For avoiding the complexity of the nonlinearity of the charging function, Montoya et al. (2017) used a piecewise linear approximation for the charging function for a VRP. Figure 1 shows an example of a piecewise linear approximation for a CS of $22 \mathrm{~kW}$ charging an EV equipped with a battery of $16 \mathrm{kWh}$. The coordinates on the Figure 1 indicate the breakpoint of the piecewise linear approximation.

\subsection{Battery degradation}

In general, most of the batteries used in electric mobility suffer a degradation process. This process causes a reduction of the energy storage capacity of the battery. Therefore, the value of the battery decreases affecting the total value of the vehicle, given that the cost of the battery is around 40\% of the EV (Bloomberg New Energy Finance, 2017). Technically, there are two conditions of the battery degradation: the calendar and cycle aging (Barré et al., 2013). The former is related to the storage conditions and pass of time; which, in many cases, is 


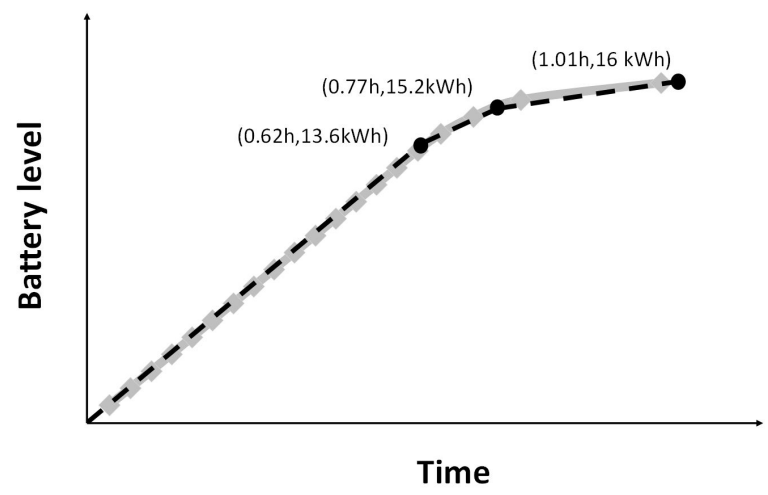

Figure 1. Real data vs. piecewise linear approximation for a CS with $22 \mathrm{~kW}$ charging a battery of $16 \mathrm{kWh}$.

unmanageable. The latter is directly related to the use conditions of the battery, and how it is discharged and charged (i.e. the energy level, the temperature, the current, among others).

Considering that the cycle aging depends on the uses of the battery, we included this component into the EBCP. In the literature there are some battery degradation models based on the electrochemical components. However, as Pelletier et al. (2017) mentioned, most of those models are not very likely to be implemented in transportation planning operations. Han et al. (2014) proposed a practical model for estimating the battery degradation based on experimental results. The authors estimated the number of cycles until the battery capacity fades down to 80\%, for different Depths of Discharge (DoD). Using those data, they estimated a wear cost function for the battery degradation process when it is discharged and charged within different intervals of the battery levels. For example, a $3 \mathrm{kWh}$ battery could be divided in three intervals of $1 \mathrm{kWh}$ each. In this way, three intervals are obtained: 0 to $1 \mathrm{kWh}, 1$ to $2 \mathrm{kWh}$, and 2 to $3 \mathrm{kWh}$. Then, each interval will have a different degradation cost related; in their study Han et al. (2014) divided the battery capacity in 10 intervals.

It is important to mention that Han et al. (2014) did not include the impact of the power in the degradation cost, since they used a domestic charger to retrieve the data. However, the powers can affect the battery degradation (Omar et al., 2014; Vutetakis \& Wu, 1992). To the best of our knowledge, there is not a battery degradation model applicable to the transportation problem that includes, simultaneously, both cyclic aging and the impact of power on the battery lifespan. For this reason, in this study we use the approach proposed by Han et al. (2014) penalizing the cost when the EB is charged using higher powers. The parameters for the penalization are estimated using the data from Omar et al. (2014). In that study, the authors estimated the reduction of the number of battery cycles using different powers with respect to a domestic power (i.e., the lowest power). Using their data, we establish a number of cycles reduction percentage for tested powers. For estimating the degradation cost for powers greater than the domestic power, we divide the cost of each interval (estimated using a domestic power) by the number of cycles reduction percentage. We are aware that this approach for including the impact of the power has limitations as we integrated two approaches with a different backgrounds; nonetheless, our intention is just to include the impact of charging the EB when using different powers.

\section{The electric boat charging problem}

\subsection{Problem description}

Let $S=\{1,2, . ., n\}$ be a set of segments that compose the route (i.e., the river). The set $S$ is composed by two subsets $S_{c s}$ and $S_{w c s}$, where each segment $s \in S_{c s}$ has a CS at the end of the segment and, otherwise, each segment $s \in S_{w c s}$ does not have any CS at the end of the segment. Let $V=\left\{v_{\min }, . ., v_{\max }\right\}$ be a set of different EB speeds, where $v_{\min }$ and $v_{\max }$ is the minimum and maximum speed of the EB. Each segment $s \in S$ has a travel time $t_{s v}$ and energy consumption $e_{s v}$ for the EB speed $v \in V$. Let $H$ be a set of different powers that a CS can provide to an EB. The parameter $f_{s h}$ is equal to $l$ if the CS at the end of the segment $s \in S_{c s}$ can provide the power $h \in H$ (i.e., each CS is equipped with different powers). Each CS at the end of the segment $s \in S_{c s}$ has a charging cost $p_{s h}$ (expressed in $\$ / k W h$ ) that depends on the used power $h \in H$. Each power $h \in H$ has a nonlinear charging function. As we mentioned before the nonlinear charging function is fitted using a piecewise linear approximation. The set $B=\{0, . ., b\}$ represents the breakpoints of the piecewise linear approximation. The parameters $a_{h k}$ and 
$l_{h k}$ represent the battery level and the charging time for the breakpoint $k \in B$ of the power $h \in H$. For including the battery degradation, let $M$ be a set of intervals of the battery level. The parameter $g_{m}$ is the upper bound of the interval $m \in M$, where $g_{m-1} \leq g_{m}$ (e.g., $g_{l}=5 \mathrm{KWh}, g_{2}=10 \mathrm{KWh}, . ., g_{5}=25 \mathrm{KWh}$ ); and the parameter $\Delta=g_{m}-g_{m-1}$ is the length of the intervals. The parameter $d_{m}$ (expressed in $\$ / k W h$ ) represents the battery degradation cost when EB is discharged within the interval $m \in M$, and the parameter $c_{h m}$ (expressed in $\$ / k W h$ ) represents the battery degradation cost when EB is charged within $m \in M$ using the power $h \in H$. Considering that the degradation cost increases with the battery level, then $d_{m-l} \leq d_{m}$ and $c_{h, m-l} \leq c_{h m}$ for the interval $m \in M$ and the power $h \in H$. The EB has a battery capacity of $Q$ (expressed in $\mathrm{kWh}$ ) and a maximum time $T_{\max }$ to complete the route. It is assumed that the EB leaves the origin with a fully charged battery.

In this problem, the objective is to decide the EB speed at each segment and where/how much to charge in a way that the total cost for the route is minimized. The total cost is defined as the sum of the energy charged cost plus the battery degradation cost. Feasible solutions for the EBCP, satisfy the following conditions: the EB begins at the starting point of the first segment (i.e., the origin) and ends at the finish point of the last segment (i.e., destination); the EB arrives to the destination before the time limit; and the battery level of the EB remains between 0 and $Q$.

\subsection{Illustrative example}

For providing the reader with a better understanding of the EBCP, we provide now a small illustrative example. Figure 2 shows a numerical example of a solution for the EBCP. Figure 2a depicts a river route with a given origin and destination, which is divided in five segments. The segments are split when there is a notorious change in the shape or in the width of the river, which affects the river flow speed. The EB direction is against the river current. Each segment has an associated energy consumption (in kWh) and a travel time (in hours) depending on the speed of the EB; those values are presented for each segment in Figure 2a. A CS is located at the end of segments 2 and 4 . Each CS has associated its respective charge prices (in $\$ / k W h$ ) and its piecewise linear charging functions corresponding to the different powers available at each of them. Regarding the degradation process, Figure $2 \mathrm{~b}$ shows the degradation cost caused by battery's discharging or charging for each of the intervals displayed. The charging process has two associated degradation costs depending on the power supplied to the battery. When a domestic charger is used, we consider the same cost as for the discharge; however, for bigger powers rates, there is a penalizing factor for increasing the degradation cost, which is shown in the last column of Figure 2b. The route must be ended before 3.5 hours. Finally, the battery capacity of the $\mathrm{EB}$ is $20 \mathrm{kWh}$ and it leaves the origin fully charged.

Figure 2c depicts a feasible solution for the instance presented in Figure 2a. The chosen speeds are displayed in each segment. The energy level and the arrival time when the EB arrives to each CS are shown within the figure. In the CS located at the end of segment 2 the charge level of the EB is $8.1 \mathrm{kWh}$. For computing the degradation cost caused by the discharging, we consider the intervals of the degradation models contained within the full charge level in the starting point $(20 \mathrm{kWh})$ and the level when the EB arrives at the CS $(8.1 \mathrm{kWh})$. This means that at this point the EB has totally drained the energy corresponding to the first two intervals of the degradation model, and $1.9 \mathrm{kWh}$ of the third one. For the case of the two first intervals ( $20 \mathrm{kWh}$ to $10 \mathrm{kWh}$ ) the cost is assumed completely $(0.239 \$ / k W h \times 5 k W h+0.176 \$ / k w h \times 5 k W h=2.076 \$)$. For the fraction discharged in the third interval the cost is calculated with an interpolation; therefore, the corresponding cost is equal to $0.300 \$(0.158 \$ / \mathrm{kWh} \times 1.9 \mathrm{kWh})$. Then the degradation cost generated by discharging is equal to $2.376 \$$ at this point. At this CS the EB charges $10.94 \mathrm{kWh}$, allowing for the battery to reach a level of $19.04 \mathrm{kWh}$. Similarly, for computing the degradation cost caused by the charging of the battery, we consider the intervals of the degradation model contained within the arriving level in the CS $(8.1 \mathrm{kWh})$ and the level when the EB finishes charging $(19.04 \mathrm{kWh})$. This charge represents a degradation cost related to the first three intervals. In the third one, the EB charges $1.9 \mathrm{kWh}(8.1 \mathrm{kWh}$ to $10 \mathrm{kWh})$, which has a degradation cost of $0.158 \$ / \mathrm{kWh}$. In the second one, the EB charges $5 \mathrm{kWh}$ (10 kWh to $15 \mathrm{kWh}$ ), which has a degradation cost of $0.176 \$ / \mathrm{kWh}$. Finally, in the first one, the EB charges $4.04 \mathrm{kWh}(15 \mathrm{kWh}$ to $19.04 \mathrm{kWh})$, which has a degradation cost of $0.239 \$ / \mathrm{kWh}$. The total charge cost associated to the battery degradation at this CS is 2.147 \$. The degradation cost for the rest of the route is calculated following the same structure, considering the discharge due to the travel in the remaining segments and the charging at the CS located at the end of segment 4. It is important to notice that the EB charges using a the faster charged at the CS at the end of the segment 4; thus, the EB incurs in a higher cost for both, charge and degradation costs. The total cost of this route is $18.163 \$$, having $3.331 \$(18.34 \%)$ associated to the degradation cost caused by the discharging of the battery, $6.699 \$(36.88 \%)$ associated to the degradation cost caused by the charging of the battery, and the remaining $8.133 \$(44.77 \%)$ associated to the cost of the charging itself. 


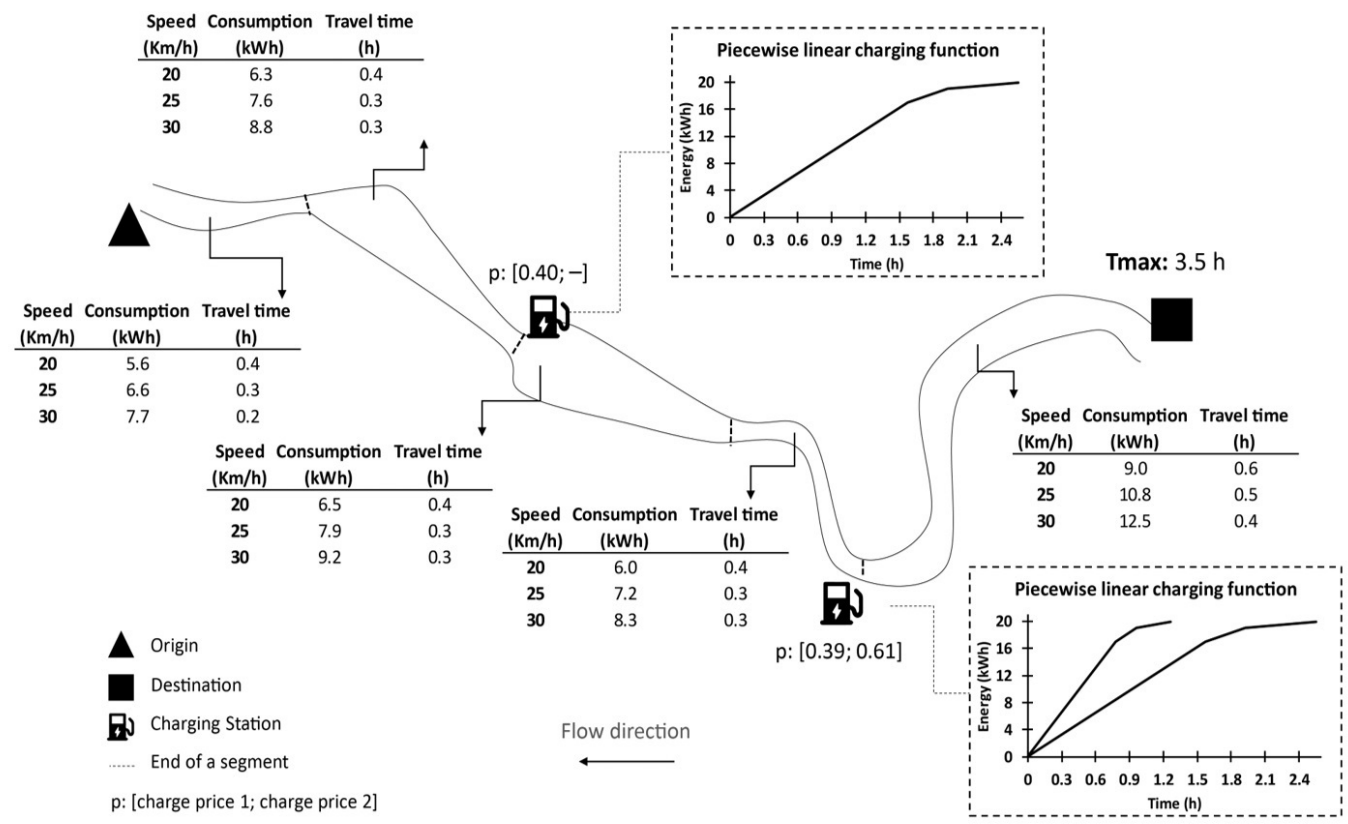

a) Problem description for a particular EBCP

\begin{tabular}{cccc} 
SoC Interval & Discharge & \multicolumn{2}{c}{ Charge } \\
(kWh) & Power 1 & Power 2 \\
\hline $\mathbf{1 5}-\mathbf{2 0}$ & 0.239 & 0.239 & 0.298 \\
$\mathbf{1 0 - 1 5}$ & 0.176 & 0.176 & 0.219 \\
$\mathbf{5}-\mathbf{1 0}$ & 0.158 & 0.158 & 0.197 \\
$\mathbf{0}-\mathbf{5}$ & 0.147 & 0.147 & 0.183
\end{tabular}

b) Battery degradation costs

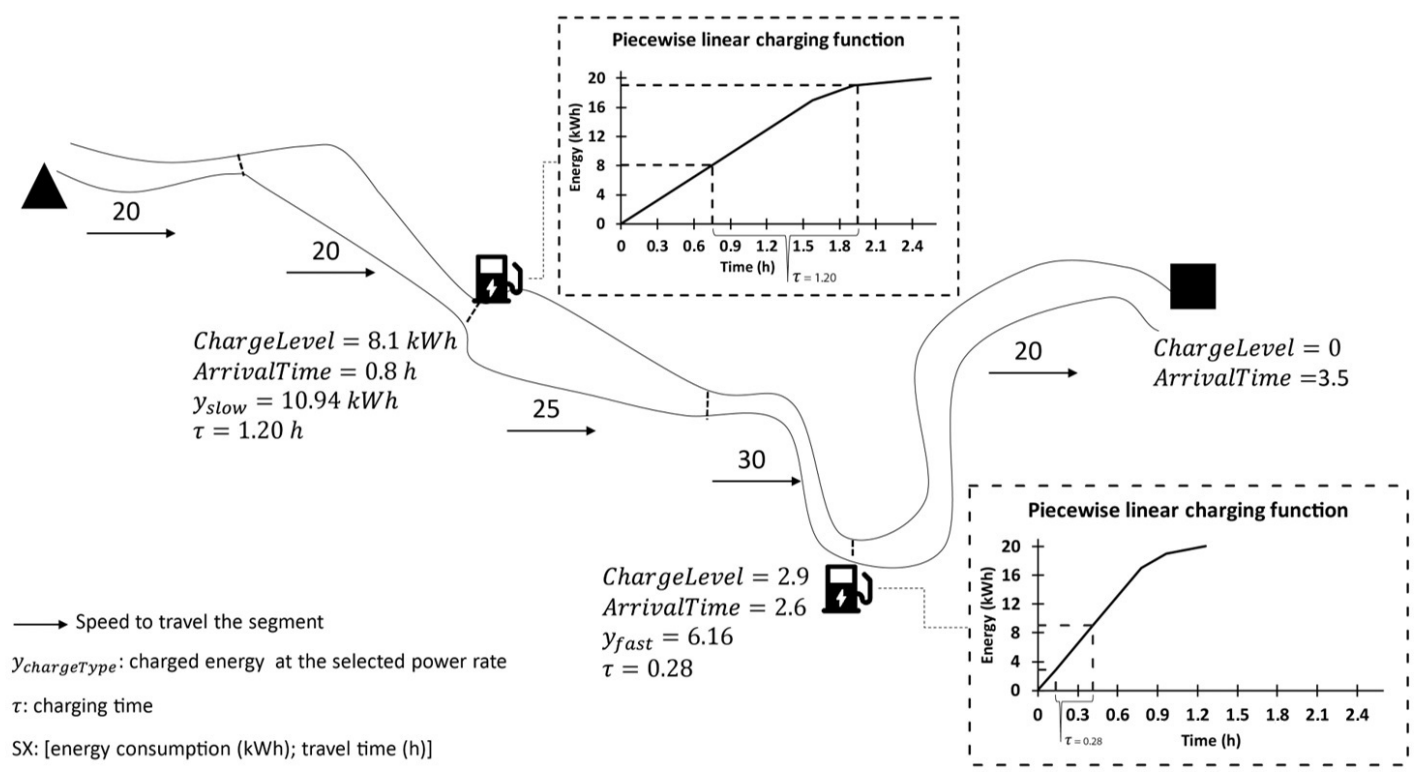

c) A solution of a particular EBCP

Figure 2. Example of a feasible solution for an EBCP. 
For estimating the stay time at each CS represented by $\tau_{s}$ we use a piecewise linear function approximation. When the EB arrives to the CS located at the end of segment 2, its battery level is $8.1 \mathrm{kWh}$ and it leaves the CS with a battery level of $19.04 \mathrm{kWh}$. Therefore, using the piecewise linear charging function, the charging time is $\tau_{2}=1.2 \mathrm{~h}$. Similarly, it is estimated the charging time when the EB arrives to the CS located at the end of segment 4, which is $\tau_{4}=0.28 \mathrm{~h}$. The total travel time is $2.02 \mathrm{~h}$. Therefore, the total time of the route is $3.5 \mathrm{~h}$, which does not exceed the value given by the $T_{\max }$ ( 3.5 hours).

\subsection{Mixed-integer linear programming formulation}

The MILP formulation uses the following decision variables: variable $\phi_{s}$ tracks the battery level when the EB finishes the segment $s \in S$. Variable $w_{s v}$ is equal to $l$ if the EB travels at the segment $s \in S$ at the speed $v \in V$; and 0 otherwise. Variable $x_{s h}$ is equal to $l$ if the EB charges at the CS located at the end of the segment $s \in S_{c s}$ using the power $h \in H$; and 0 otherwise. Variables $y_{s h}$ and $\tau_{s}$ represents the amount of energy and the charging time when the EB charges at the CS located at the end of the segment $s \in S_{c s}$. Variables $o_{s}$ and $r_{s}$ specify the charging times when the EB arrives at and departs from a CS located at the end of segment $s \in S_{c s}$. Variables $z_{s h k}$ and $u_{s h k}$ are equal to 1 if the charging level is between the breakpoints $a_{h k}$ and $a_{h, k+1}$ with $k \in B \backslash\{b\}$ when the EB arrives at and departs from the CS located at the end of the segment $s \in S_{c s}$, and the EB is charged using the power $h \in H$; and 0 otherwise. Variables $\alpha_{\text {shk }}$ and $\lambda_{\text {shk }}$ are the coefficient of the breakpoint $k \in B$ in the piecewise linear approximation, when the EB is charged using the power $h \in H$ at the CS located at the end of the segment $s \in S_{c s}$. Variable $\pi_{s m}$ is equal to 1 if the EB is discharged in the interval $m \in M$ when traveling in the segment $s \in S$. Variable $\gamma_{s m}$ is equal to 1 if the EB is charged in the interval $m \in M$ in the CS located at the end of the segment $s \in S_{c s}$. Variable $\mu_{s m}$ determines the amount of discharged energy in the interval $m \in M$, when the EB travels across the segment $s \in S$. Finally, variable $\psi_{\text {shm }}$ determines the amount of charged energy in the interval $m \in M$ at the CS at the end of the segment $S_{c s}$, when the EB is charged using a power of $h \in H$.

$$
\operatorname{Min} \sum_{s \in S_{c s}} \sum_{h \in H} p_{s h} y_{s h}+\sum_{s \in S} \sum_{m \in M} d_{m} \mu_{s m}+\sum_{s \in S_{c s}} \sum_{h \in H} \sum_{m \in M} c_{h m} \psi_{s h m}
$$

Subject to

$$
\begin{gathered}
\sum_{v \in V} w_{s v}=1, \quad \forall s \in S \\
\phi_{1}=Q-\sum_{v \in V} e_{l v} w_{l v}, \\
\phi_{s}=\phi_{s-1}-\sum_{v \in V} e_{s v} w_{s v}, \quad \forall s \in S: s>1 \Lambda s-1 \in S_{w c s} \\
\phi_{s}=\phi_{s-I}+\sum_{h \in H} y_{s-1, h}-\sum_{v \in V} e_{s v} w_{s v}, \quad \forall s \in S: s>1 \Lambda s-1 \in S_{c s} \\
\phi_{s}+\sum_{h \in H} y_{s h} \leq Q, \quad \forall s \in S_{c s} \\
y_{s h} \leq x_{s h} Q, \quad \forall s \in S_{c s}, \forall h \in H \\
\sum_{h \in H} x_{s h} \leq 1, \quad \forall s \in S_{c s} \\
x_{s h} \leq f_{s h}, \quad \forall s \in S_{c s}, \forall h \in H \\
\sum_{v \in V} \sum_{s \in S} t_{s v} w_{s v}+\sum_{s \in S_{c s}} \tau_{s} \leq T_{\max }, \\
\phi_{s}=\sum_{h \in H} \sum_{k \in B} \alpha_{s h k} a_{h k}, \quad \forall s \in S_{c s} \\
o_{s}=\sum_{h \in H} \sum_{k \in B} \alpha_{s h k} l_{h k}, \quad \forall s \in S_{c s} \\
\sum_{k \in B} \alpha_{s h k}=\sum_{k \in B} z_{s h k}, \quad \forall s \in S_{c s}, \forall h \in H
\end{gathered}
$$




$$
\begin{aligned}
& \sum_{k \in B} z_{s h k} \leq x_{s h}, \quad \forall s \in S_{c s}, \forall h \in H \\
& \alpha_{s h 0} \leq z_{s h 0}, \quad \forall s \in S_{c s}, \forall h \in H \\
& \alpha_{s h k} \leq z_{s h k}+z_{s h, k-1}, \quad \forall s \in S_{c s}, \forall h \in H, \forall k \in B \backslash\{0, b\} \\
& \alpha_{s h b} \leq z_{s h, b-1} \quad \forall s \in S_{c s}, \forall h \in H \\
& \phi_{s}+\sum_{h \in H} y_{s h}=\sum_{h \in H} \sum_{k \in B} \lambda_{s h k} a_{h k}, \quad \forall s \in S_{c s} \\
& r_{s}=\sum_{h \in H} \sum_{k \in B} \lambda_{s h k} l_{h k}, \quad \forall s \in S_{c s} \\
& \sum_{k \in B} \lambda_{s h k}=\sum_{k \in B} u_{s h k}, \quad \forall s \in S_{c s}, \forall h \in H \\
& \sum_{k \in B} u_{s h k} \leq x_{s h}, \quad \forall s \in S_{c s}, \forall h \in H \\
& \lambda_{s h 0} \leq u_{s h 0} \quad \forall s \in S_{c s}, \forall h \in H \\
& \lambda_{s h k} \leq u_{s h k}+u_{s h, k-1}, \quad \forall s \in S_{c s}, \forall h \in H, \forall b \in B \backslash\{0, b\} \\
& \lambda_{s h b} \leq u_{s h, b-1}, \quad \forall s \in S_{c s}, \forall h \in H \\
& \tau_{s}=r_{s}-o_{s}, \quad \forall s \in S_{c s} \\
& \sum_{m \in M} \mu_{s m}=\sum_{v \in V} e_{s v} w_{s v}, \quad \forall s \in S \\
& \mu_{s m} \leq \Delta \pi_{s m} \quad \forall s \in S, \forall m \in M
\end{aligned}
$$$$
\mu_{s m} \leq g_{m}-\phi_{s}+\left(1-\pi_{s m}\right) Q \quad \forall s \in S, \forall m \in M
$$$$
\sum_{m \in M} \psi_{s h m}=y_{s h}, \quad \forall s \in S_{c s}, \forall h \in H
$$$$
\sum_{h \in H} \psi_{s h m} \leq \Delta \gamma_{s m} \quad \forall s \in S_{c s}, \forall m \in M
$$$$
\sum_{h \in H} \psi_{s h m} \leq g_{m}-\phi_{S}+\left(1-\gamma_{s m}\right) Q \quad \forall s \in S_{c s}, \forall m \in M
$$$$
x_{s h} \in\{0,1\} \quad \forall s \in S_{c s}, \forall h \in H
$$$$
w_{s v} \in\{0,1\} \quad \forall s \in S, \forall v \in V
$$$$
z_{\text {shk }}, u_{\text {shk }} \in\{0,1\} \quad \forall s \in S_{c s}, \forall h \in H, \forall k \in B \backslash\{b\}
$$$$
\pi_{s m} \in\{0,1\} \quad \forall s \in S, \forall m \in M
$$$$
\gamma_{s m} \in\{0,1\} \quad \forall s \in S_{c s}, \forall m \in M
$$$$
\phi_{s} \geq 0 \quad \forall s \in S
$$ 


$$
\begin{gathered}
o_{s}, r_{s}, \tau_{s} \geq 0 \quad \forall s \in S_{c s} \\
y_{s h} \geq 0 \quad \forall s \in S_{c s}, \forall h \in H \\
\mu_{s m} \geq 0 \quad \forall s \in S, \forall m \in M \\
\alpha_{s h k}, \lambda_{s h k} \geq 0 \quad \forall s \in S_{c s}, \forall h \in H, \forall k \in B \\
\psi_{s h m} \geq 0 \quad \forall s \in S_{c s}, \forall h \in H, \forall m \in M
\end{gathered}
$$

The objective function 1 seeks to minimize the total costs (degradation cost plus charging cost). Constraints 2 ensure that only one speed is selected for traveling across each segment. Constraints 3-5 track the battery level at the end of each segment. Constraints 6 ensure that the energy level is bounded by the battery capacity. Constraints 7 impose that the EB only charges at the CS selected for the charging. Constraints 8 impose that only one power can be selected to charge the EB. Constraints 9 ensure that the EB can charge only with a power $h \in H$ available at the CS located at the end of the segment $s \in S_{c s}$. Constraint 10 ensures that the EB completes the route no later than $T_{\max }$. Constraints 11-17 define the charge level (and its corresponding charging time) when the EB arrives at the CS located at the end of the segment $s \in S_{c s}$. Similarly, Constraints 18-24 define the charge level (and its corresponding charging time) when the EB departs from the CS located at the end of the segment $s \in S_{c s}$. Constraints 25 define the time spent at any CS. Constraints 26-28 determine the amount of discharged energy for each segment $s \in S$ and for each interval $m \in M$. Similarly, Constraints 29-31 determine the amount of charged energy for each interval $m \in M$, at the CS located at the end of the segment $s \in S_{c s}$. Finally, Constraints 32-42 define the domain of the decision variables.

\section{Computational experiments}

For testing the performance of the MILP formulation for the EBCP, we used a set of instances based on a real river transport operation. We focus on presenting the capability of the MILP to optimally solve different instances within reasonable CPU time. Additionally, we performed a sensitivity analysis of the results from the MILP formulation under different conditions.

\subsection{Test instances for the EBCP}

The research project Energética 2030 (lcetex, 2017) pretends to implement a river transport operation with EBs in the Magdalena river (lower Magdalena zone). This implementation has a limited scope (maximum two CSs), but it is expected to expand the operation with more CSs. Considering that one key for the successful implementation of the electric mobility is having tools for making smart charging decisions, this project includes the development of the optimization models for the planning of the river transport operation. For this reason, the instances used in this paper were built considering the future implementation of river transport operation with EBs in the lower Magdalena influence. The set comprises 20 instances, which were based on different origins and destinations in the zone. We assume that the CSs will be located in settlements or cities located in intermediate points between the origin and the destination with access to the electric service.

For generating the data presented in the instances, we used different approaches. Energy consumption is generated with the Crouch's formula presented in Minami \& Yamachika, (2004). The time needed to travel between segments considers the length of each segment, the river speed, and the different speeds of the EB. For estimating the energy consumption and travel time, we used a speed interval of $1 \mathrm{~km} / \mathrm{h}$ (e.g., $V=\{5,6,7,8,9,10\})$. The battery capacities were selected considering the distance of the segments and the EB constraints, and $T_{\max }$ was selected considering the route constraints. For the charging cost at each CS we took as a reference the costs of the Blink network (Blink Charging, 2018). For determining the degradation cost, first, it is necessary to estimate the cost of the battery pack. We based on the information presented in Battery University, (2017) and Union of Concerned Scientists, (2018) for estimating this value. Having this cost, we determined the battery degradation cost with the model presented in Han et al. (2014). For determining the penalizing of the degradation costs when the high power rates are used, we took as reference the approach presented in Omar et al. (2014). 
We generate 6 additional instances for each of the 20 original instances (for a total of 140 instances) varying different elements: $T_{\max }$, battery capacity, and the magnitude of the speed discretization. The $T_{\max }$ is modified by reducing its original value in $10 \%$ and $20 \%$. Similarly, the battery capacity is modified by reducing its original value in $10 \%$ and $20 \%$. Finally, the speed discretization is set to 0.5 and $0.25 \mathrm{~km} / \mathrm{h}$. For each scenario, we only modified the value that we wanted to evaluate and kept the remaining ones fixed.

\subsection{Experimental environment}

Gurobi Optimizer (version 8.1.0) was used to solve the MILP formulation of the EBCP, with a CPU time limit of 10,800s. We ran all the experiments on a computer with Intel Xeon E5-2670 processor (with 16 cores at $2.6 \mathrm{GHz}$ ) and $64 \mathrm{~GB}$ of RAM running on linux CentOS 6.6

\subsection{Results of the MILP formulation for the EBCP}

Table 1 shows the detailed results of the 20 original EBCP instances using the proposed MILP formulation. Column 1 lists the instances names. Columns 2 to 4 presents the characteristics of the instances (segments, number of CSs, and total length). Column 5 details the objective function value. Column 6 presents the optimality gap. Column 7 details the CPU time (in seconds). Additionally, for comparing the cost of the EB against 1CB, we estimated the cost of the routes when we used an ICB, this is shown in Column 8. For converting the required energy to fuel consumption, we used the data presented by lee (2013); and for estimating the cost, we used a fuel price of USD 3.13, which is the standard price in the influence zone. Column 9 presents the gap (in \%) between the EB vs ICB cost. It is important to notice that ICB cost only accounts the fuel cost; however, ICBs have a related Social Cost of Carbon (SCC). For including the SCC, we first estimated the $\mathrm{Kg}$ of $\mathrm{CO}_{2}$ emissions (column 10) using the information of Sailors for the Sea (2019); and with this information we estimated the SCC (column 11) using the information presented by Nordhaus (2017). Column 12 shows the combined costs for the ICB (refueling costs + SCC). Finally, column 13 details the cost gap (in \%) of the EB vs ICB.

The results in Table 1 show that the MILP is able to optimally solve all the original instances (20/20). The average CPU time is $4.77 \mathrm{~s}$ and the maximum value $82.5 \mathrm{~s}$. The results show that our MlLP formulation is able to find optimal solutions in competitive CPU times for an operative planning problem with EBs.

Table 1. Results of the EBCP base instances.

\begin{tabular}{|c|c|c|c|c|c|c|c|c|c|c|c|c|}
\hline Instance & $\begin{array}{c}\text { \# of } \\
\text { segments }\end{array}$ & \# of CS & $\begin{array}{c}\text { Total } \\
\text { length } \\
(\mathrm{km})\end{array}$ & $\begin{array}{l}\text { Objective } \\
\text { Function }\end{array}$ & $\begin{array}{l}\text { Optimality } \\
\text { Gap }\end{array}$ & $\begin{array}{c}\text { CPU } \\
\text { time (s) }\end{array}$ & $\begin{array}{c}\text { Cost } \\
\text { with } \\
\text { fuel }(\$)\end{array}$ & Gap (\%) & $\begin{array}{c}\mathrm{CO}_{2} \\
\text { emissions } \\
(\mathrm{Kg})\end{array}$ & SCC (\$) & $\begin{array}{c}\text { Total } \\
\text { cost }(\$)\end{array}$ & Gap (\%) \\
\hline 1 & 17 & 2 & 124.2 & 45.7 & $0.01 \%$ & 0.4 & 38.9 & $14.8 \%$ & 119.6 & 8.0 & 46.9 & $-2.7 \%$ \\
\hline 2 & 23 & 2 & 144.8 & 55.7 & $0.01 \%$ & 0.5 & 47.8 & $14.2 \%$ & 146.9 & 9.8 & 57.6 & $-3.5 \%$ \\
\hline 3 & 16 & 1 & 84.8 & 25.8 & $0.00 \%$ & 0.1 & 30.2 & $-16.7 \%$ & 92.7 & 6.2 & 36.4 & $-40.8 \%$ \\
\hline 4 & 32 & 3 & 174.1 & 96.8 & $0.01 \%$ & 1.0 & 64.1 & $33.8 \%$ & 197.0 & 13.2 & 77.3 & $20.2 \%$ \\
\hline 5 & 35 & 4 & 248.4 & 60.9 & $0.01 \%$ & 0.6 & 51.1 & $16.1 \%$ & 157.2 & 10.5 & 61.6 & $-1.2 \%$ \\
\hline 6 & 46 & 4 & 289.5 & 109.3 & $0.00 \%$ & 1.8 & 65.5 & $40.1 \%$ & 201.3 & 13.5 & 79.0 & $27.7 \%$ \\
\hline 7 & 32 & 2 & 169.7 & 33.4 & $0.00 \%$ & 0.4 & 34.4 & $-3.1 \%$ & 105.8 & 7.1 & 41.5 & $-24.3 \%$ \\
\hline 8 & 64 & 6 & 348.2 & 157.7 & $0.01 \%$ & 82.5 & 84.1 & $46.7 \%$ & 258.6 & 17.3 & 101.4 & $35.7 \%$ \\
\hline 9 & 23 & 2 & 73.2 & 24.1 & $0.01 \%$ & 1.0 & 26.6 & $-10.2 \%$ & 81.8 & 5.5 & 32.1 & $-32.9 \%$ \\
\hline 10 & 20 & 3 & 114.4 & 51.1 & $0.01 \%$ & 0.4 & 45.3 & $11.4 \%$ & 139.3 & 9.3 & 54.6 & $-6.9 \%$ \\
\hline 11 & 20 & 1 & 112.0 & 35.2 & $0.01 \%$ & 0.2 & 40.5 & $-15.0 \%$ & 124.4 & 8.3 & 48.8 & $-38.6 \%$ \\
\hline 12 & 20 & 2 & 134.6 & 65.2 & $0.00 \%$ & 0.3 & 46.9 & $28.0 \%$ & 144.3 & 9.7 & 56.6 & $13.1 \%$ \\
\hline 13 & 18 & 1 & 114.0 & 41.5 & $0.00 \%$ & 0.3 & 41.3 & $0.5 \%$ & 127.0 & 8.5 & 49.8 & $-20.0 \%$ \\
\hline 14 & 14 & 1 & 97.5 & 31.9 & $0.00 \%$ & 0.1 & 34.8 & $-9.2 \%$ & 107.2 & 7.2 & 42.0 & $-31.7 \%$ \\
\hline 15 & 46 & 4 & 146.3 & 13.9 & $0.00 \%$ & 0.9 & 27.8 & $-99.2 \%$ & 85.4 & 5.7 & 33.5 & $-140.3 \%$ \\
\hline 16 & 40 & 6 & 228.7 & 52.2 & $0.01 \%$ & 2.9 & 49.9 & $4.4 \%$ & 153.4 & 10.3 & 60.2 & $-15.3 \%$ \\
\hline 17 & 40 & 2 & 224.1 & 39.0 & $0.00 \%$ & 0.3 & 42.7 & $-9.5 \%$ & 131.4 & 8.8 & 51.5 & $-32.1 \%$ \\
\hline 18 & 40 & 4 & 269.2 & 64.0 & $0.01 \%$ & 1.2 & 53.2 & $16.8 \%$ & 163.7 & 11.0 & 64.2 & $-0.4 \%$ \\
\hline 19 & 36 & 2 & 228.0 & 47.0 & $0.01 \%$ & 0.4 & 44.4 & $5.5 \%$ & 136.5 & 9.1 & 53.5 & $-14.0 \%$ \\
\hline 20 & 28 & 2 & 195.0 & 33.8 & $0.00 \%$ & 0.2 & 38.1 & $-12.7 \%$ & 117.1 & 7.8 & 45.9 & $-35.9 \%$ \\
\hline $\begin{array}{l}\text { Average } \\
\text { results }\end{array}$ & & 2.7 & 176.0 & 54.2 & $0.00 \%$ & 4.8 & 45.4 & $2.8 \%$ & 139.5 & 9.3 & 54.7 & $-17.2 \%$ \\
\hline
\end{tabular}


When the operation is executed with an EB, there is an average gap of $2.8 \%$ with respect to the operation with an ICB, only considering fuel costs. However, if we include the associated SCC to the operation with an ICB, there is an average reduction of $17 \%$ in the average gap. In this way, SCC is an important element to consider when comparing electric vehicles with internal combustion vehicles since it highlights the environmental benefits of clean energies. Furthermore, it is important to note that in the cases where the cost of the EB operation was superior to the ICB operation, the average proportion of energy charged using fast chargers was around $80 \%$. This demonstrates the impact of fast chargers in the cost. It is important to notice that the fast charge impacts both charge costs and battery degradation costs. For this reason, one way to reduce the cost when using EBs is to increase the time limit $\left(T_{\max }\right)$ because this would reduce the use of fast chargers.

\subsection{Sensitivity analysis}

We now provide a sensitivity analysis to test how the variation in some of the elements that compose the instances can affect the performance of the model and the decision-making. Firstly, we consider the driving range because it is one of the main restrictions when using EVs. We pose the hypothesis that a reduction in the battery capacity of the EB can seriously affect not only its autonomy, but also the charging decisions. This could generate more frequent visits to the CSs, and probably, change the speed decisions. In addition, we consider that a reduction on the time limit has an important impact on the speed decisions, and thus, in the charging decisions. This in some cases leads to the use of fast chargers to keep the feasibility by $T_{\max }$. Furthermore, we evaluate how the smaller discretization of the speed values affects the performance of the model.

\subsubsection{Battery capacity reduction}

Table 2 shows the variation on the objective function and different type of decisions when a reduction of $10 \%$ and $20 \%$ is made in the battery capacity. When there is a reduction on the battery capacity of $10 \%$, the MILP is capable to optimally solve $20 / 20$ instances. However, with a $20 \%$, reduction, one of the instances becomes infeasible and 19/20 instances are optimally solved (row 1). Row 2 shows that a reduction on the capacity generates an increase on the objective function around 7\% and 10\%. Analysing the cost composition, we found that the degradation and energy cost increase more than 10\% (rows 3 and 4). This is expected, because with a smaller battery the EB needs to charge more often. Moreover, smaller battery are cheaper than the original battery, for that reason the battery degradation cost in discharge decreases in both scenarios (row 5). Rows 6-9 present the charging decisions about slow or fast charges and number of charges for each scenario. Finally, the capacity reduction affects not only the charging decisions, but also speed decisions, because the reduction on the autonomy. Row 10 shows an increase of the average speed for both scenarios.

Table 2. Variation in the objective function and the decisions caused by $10 \%$ and $20 \%$ battery capacity reduction.

\begin{tabular}{|c|c|c|c|c|c|}
\hline Parameter & Original & $\Delta 10 \%$ & Gap (\%) & $\Delta 20 \%$ & Gap (\%) \\
\hline Feasible solutions & $20 / 20$ & $20 / 20$ & - & $19 / 20$ & - \\
\hline Avg. Obj. Function (\$) & 54.2 & 57.8 & $6.7 \%$ & 59.5 & $9.7 \%$ \\
\hline Avg. Charged energy cost (\$) & 24.6 & 27.5 & $11.7 \%$ & 29.6 & $20.3 \%$ \\
\hline $\begin{array}{l}\text { Avg. degradation cost (\$) } \\
\text { (in charge) }\end{array}$ & 8.3 & 9.4 & $13.7 \%$ & 10.2 & $23.2 \%$ \\
\hline $\begin{array}{l}\text { Avg. degradation cost (\$) } \\
\text { (in discharge) }\end{array}$ & 21.3 & 20.9 & $-1.9 \%$ & 19.7 & $-7.8 \%$ \\
\hline $\begin{array}{l}\text { Avg. Charged energy (kWh) } \\
\text { (Slow charge) }\end{array}$ & 29.5 & 33.6 & $13.9 \%$ & 31.0 & $4.9 \%$ \\
\hline $\begin{array}{l}\text { Avg. Charged energy (kWh) } \\
\text { (Fast charger) }\end{array}$ & 21.7 & 23.8 & $9.5 \%$ & 30.9 & $42.2 \%$ \\
\hline $\begin{array}{l}\text { Avg. \# of charges } \\
\text { (Slow Charger) }\end{array}$ & 1.5 & 1.6 & $6.7 \%$ & 1.3 & $-12.3 \%$ \\
\hline $\begin{array}{l}\text { Avg. \# of charges } \\
\text { (Fast charger) }\end{array}$ & 0.9 & 1.0 & $11.8 \%$ & 1.2 & $36.2 \%$ \\
\hline Average speed $(\mathrm{Km} / \mathrm{h})$ & 12.4 & 12.7 & $2.2 \%$ & 12.8 & $3.1 \%$ \\
\hline
\end{tabular}




\subsubsection{Time limit reduction}

Table 3 shows how the objective function and charging and speed decisions variates with a reduction of $10 \%$ and $20 \%$ of $T_{\max }$. When there is a reduction on the time limit of $10 \%$, the MILP is capable to optimally solve 20/20 instances. However, when the reduction is 20\%, two of the instances become infeasible and $18 / 20$ instances are optimally solved (row 1 ). Row 2 shows that a reduction on the battery capacity generates an increasing on the objective function of about 17\% and 11\%. Analyzing the cost composition (rows 3-4), we found the degradation cost (in charge) and energy cost increase at least 5\%. Although the use of slow chargers decreases, the use of fast chargers has an abrupt increase (rows 6 - 9), which justify the cost growing. Finally, $T_{\max }$ impacts the speed decisions as expected. Row 10 shows an increase of the average speed for both scenarios.

Table 3. Variation in the objective function and the decisions caused by $10 \%$ and $20 \%$ Tmax reduction.

\begin{tabular}{|c|c|c|c|c|c|}
\hline Parameter & Base & $\Delta 10 \%$ & Gap (\%) & $\Delta 20 \%$ & Gap $(\%)$ \\
\hline Feasible solutions & $20 / 20$ & $20 / 20$ & - & $18 / 20$ & - \\
\hline Avg. Obj. Function (\$) & 54.2 & 63.2 & $16.5 \%$ & 60.3 & $11.2 \%$ \\
\hline Avg. Charged energy cost (\$) & 24.6 & 31.1 & $26.3 \%$ & 29.9 & $21.7 \%$ \\
\hline $\begin{array}{l}\text { Avg. degradation cost (\$) } \\
\text { (in charge) }\end{array}$ & 8.3 & 9.6 & $16.5 \%$ & 8.8 & $6.8 \%$ \\
\hline $\begin{array}{l}\text { Avg. degradation cost (\$) } \\
\text { (in discharge) }\end{array}$ & 21.3 & 22.5 & $5.3 \%$ & 21.5 & $0.8 \%$ \\
\hline $\begin{array}{l}\text { Avg. Charged energy (kWh) } \\
\text { (Slow charge) }\end{array}$ & 29.5 & 18.8 & $-36.2 \%$ & 7.8 & $-73.7 \%$ \\
\hline $\begin{array}{l}\text { Avg. Charged energy (kWh) } \\
\text { (Fast charger) }\end{array}$ & 21.7 & 39.5 & $81.8 \%$ & 44.9 & $106.5 \%$ \\
\hline $\begin{array}{l}\text { Avg. \# of charges } \\
\text { (Slow Charger) }\end{array}$ & 1.5 & 1.1 & $-30.0 \%$ & 0.6 & $-59.3 \%$ \\
\hline $\begin{array}{l}\text { Avg. \# of charges } \\
\text { (Fast charger) }\end{array}$ & 0.9 & 1.4 & $64.7 \%$ & 1.6 & $83.0 \%$ \\
\hline Average speed $(\mathrm{Km} / \mathrm{h})$ & 12.4 & 13.5 & $9.0 \%$ & 14.5 & $16.9 \%$ \\
\hline
\end{tabular}

\subsubsection{Variation on the speed discretization}

As we mentioned in the problem description, there is a set $V$ of possible speeds. In the original instances, the set $V$ is discretized with a speed interval of $1 \mathrm{~km} / \mathrm{h}$. Table 4 presents the results of the instances when the speed interval takes values of 0.5 and $0.25 \mathrm{~km} / \mathrm{h}$. The results show that changing the discretization intervals of the speed do not generate an important change on the objective function value. In average, an improvement of only $0.1 \%$ is reached (row 1 ). The max gap is around $0.2 \%$, which is negligible (row 2). Finally, even though the average time is negligible for all the cases, there is an important increase in the CPU time for both scenarios.

Table 4. Changes on objective function caused by variations on the speed interval 0.5 and $0.25 \mathrm{~km} / \mathrm{h}$.

\begin{tabular}{lccc}
\hline & $1(\mathrm{Km} / \mathrm{h})$ & $0.5(\mathrm{Km} / \mathrm{h})$ & $0.25(\mathrm{Km} / \mathrm{h})$ \\
\hline Avg. Gap (\%) & & $0.08 \%$ & $0.10 \%$ \\
Max Gap (\%) & & $0.17 \%$ & $0.18 \%$ \\
CPU time (s) & 4.77 & 5.62 & 7.03 \\
\hline
\end{tabular}

\section{Conclusions}

This paper introduces a new optimization problem called the Electric Boat Charging Problem (EBCP). This problem is based on the application of electric mobility in a river transport operation problem. For solving this problem, we propose a MILP formulation. For evaluating the performance of the MILP formulation, we used a set of instances based on a future river transport operation located in the Magdalena river, Colombia. The pilot test is part of the research project "Energética 2030". The results show that the MILP formulation is capable to optimally solve 20/20 instances in competitive CPU times.

We made a sensitive analysis for testing how variations in different elements of the problem affect its performance. Battery capacity reduction shows an important increase in the total cost. The $T_{\max }$ appears to be the most critical element, since a reduction of 10\% and 20\% represented an increase of more than $11 \%$ in the 
average total costs. The $T_{\max }$ reduction leads to a variation on the speed and the charging decisions, which affects the use of fast chargers. Finally, a variation in the speed interval does not significantly impact the objective function. We can conclude that both battery capacity and time limit $\left(T_{\max }\right)$ are critical elements. For that reason, these two parameters should be considered, when designing a river transport operation with EBs.

\section{Acknowledgements}

The authors would like to thank Universidad EAFIT for supporting this research through the Research Assistantship grant from project 828-000068. This research has also been developed in the framework of the "ENERGETICA 2030" Research Program, with code 58667 in the "Scientific Colombia" initiative, funded by The World Bank through the call "778-2017 Scientific Ecosystems", managed by the Colombian Administrative Department of Science, Technology and Innovation (COLCIENCIAS). We would also like to thank Evelin Cañola for her assistance in the validation of the model features. Additionally, we would like to thank Universidad EAFIT scientific computing center (APOLO) for its support for the computational experiments.

\section{References}

Alvarado Ponce, L. (2017). Estudio del potencial de las embarcaciones solares en la Amazonía: caso de estudio Río Napo (Master thesis). E.T.S.l. Diseño Industrial, Universidad Politécnica de Madrid, Madrid.

Arcadis Nederland BV, JESYCA S.A.S. (2015). Plan maestro fluvial de Colombia 2015. Bogotá: Ministerio de Transporte de Colombia.

Barré, A., Deguilhem, B., Grolleau, S., Gérard, M., Suard, F., \& Riu, D. (2013). A review on lithium-ion battery ageing mechanisms and estimations for automotive applications. Journal of Power Sources, 241, 680-689. http://dx.doi.org/10.1016/j.jpowsour.2013.05.040.

Battery University. (2017). Cost of mobile and renewable power. Retrieved in 2019, May 2, from https://batteryuniversity.com/learn/ article/bu_1006_cost_of_mobile_power

Baum, M., Dibbelt, J., Hübschle-schneider, L., Pajor, T., \& Wagner, D. (2014). Speed-consumption tradeoff for electric vehicle route planning. In Proceedings of the 14th Workshop on Algorithmic Approaches for Transportation Modelling, Optimization, and Systems (pp. 138-151). Germany: Schloss Dagstuhl-Leibniz-Zentrum fuer Informatik. https://doi.org/10.4230/OASlcs.ATMOS.2014.138.

Bektaş, T., \& Laporte, G. (2011). The pollution-routing problem. Transportation Research Part B: Methodological, 45(8), 1232-1250. http://dx.doi.org/10.1016/j.trb.2011.02.004.

Betancur, E., Osorio-Gómez, G., \& Rivera, J. C. (2017). Heuristic optimization for the energy management and race strategy of a solar car. Sustainability, 9(10), 1576. http://dx.doi.org/10.3390/su9101576.

Blink Charging. (2018). EV charging fees. Retrieved in 2019, June 18, from https://www.blinkcharging.com/ev-charging-fee

Bloomberg New Energy Finance. (2017). Electric cars to reach price parity by 2025. Retrieved in 2019, June 18, from https://about. bnef.com/blog/electric-cars-reach-price-parity-2025/

Fagerholt, K., Laporte, G., \& Norstad, 1. (2010). Reducing fuel emissions by optimizing speed on shipping routes. The Journal of the Operational Research Society, 61(3), 523-529. http://dx.doi.org/10.1057/jors.2009.77.

Habib, L., Bayne, E. M., \& Boutin, S. (2007). Chronic industrial noise affects pairing success and age structure of ovenbirds Seiurus aurocapilla. Journal of Applied Ecology, 44(1), 176-184. http://dx.doi.org/10.1111/j.1365-2664.2006.01234.x.

Han, S., Han, S., \& Aki, H. (2014). A practical battery wear model for electric vehicle charging applications. Applied Energy, 113, 11001108. http://dx.doi.org/10.1016/j.apenergy.2013.08.062.

lcetex. (2017). Colombia científica. Retrieved in 2019, June 18, from http://colombiacientifica.gov.co/colombia/

Jaimurzina, A., Wilmsmeier, G., \& Montiel, D. (2017). Eficiencia energética y movilidad eléctrica fluvial: soluciones sostenibles para la Amazonía. México: CEPAL.

Lee, E.-C. (2013). On the water: save fuel, money: running your boat by the numbers. Retrieved in 2019, May 30, from https://ncseagrant. ncsu.edu/coastwatch/previous-issues/2013-2/summer-2013/on-the-water-save-fuel-money-running-your-boat-by-the-numbers/

Minami, S., \& Yamachika, N. (2004). A practical theory of the performance of low velocity boat. Journal of Asian Electric Vehicles, 2(1), 535-539. http://dx.doi.org/10.4130/jaev.2.535.

Mitson, R. B. (1995). Underwater noise of research vessels (ICES Cooperative Research Report, 209). Denmark: ICES.

Montoya, A., Guéret, C., Mendoza, J. E., \& Villegas, J. G. (2017). The electric vehicle routing problem with nonlinear charging function. Transportation Research Part B: Methodological, 103, 87-110. http://dx.doi.org/10.1016/j.trb.2017.02.004.

Nordhaus, W. D. (2017). Revisiting the social cost of carbon. Proceedings of the National Academy of Sciences of the United States of America, 114(7), 1518-1523. http://dx.doi.org/10.1073/pnas.1609244114. PMid:28143934.

Norstad, 1., Fagerholt, K., \& Laporte, G. (2011). Tramp ship routing and scheduling with speed optimization. Transportation Research Part C, Emerging Technologies, 19(5), 853-865. http://dx.doi.org/10.1016/j.trc.2010.05.001.

Omar, N., Monem, M. A., Firouz, Y., Salminen, J., Smekens, J., Hegazy, O., Gaulous, H., Mulder, G., Van den Bossche, P., Coosemans, T., \& Van Mierlo, J. (2014). Lithium iron phosphate based battery: assessment of the aging parameters and development of cycle life model. Applied Energy, 113, 1575-1585. http://dx.doi.org/10.1016/j.apenergy.2013.09.003.

Pelletier, S., Jabali, O., Laporte, G., \& Veneroni, M. (2017). Battery degradation and behaviour for electric vehicles: review and numerical analyses of several models. Transportation Research Part B: Methodological, 103, 158-187. http://dx.doi.org/10.1016/j.trb.2017.01.020.

Pelletier, S., Jabali, 0., Laporte, G., Pelletier, S., Jabali, O., \& Laporte, G. (2016). 50th anniversary invited article: goods distribution with electric vehicles: review and research perspectives. Transportation Science, 50(1), 3-22. http://dx.doi.org/10.1287/trsc.2015.0646. 
Psaraftis, H. N., \& Kontovas, C. A. (2014). Ship speed optimization: concepts, models and combined speed-routing scenarios. Transportation Research Part C, Emerging Technologies, 44, 52-69. http://dx.doi.org/10.1016/j.trc.2014.03.001.

Sadeghi-Barzani, P., Rajabi-Ghahnavieh, A., \& Kazemi-Karegar, H. (2014). Optimal fast charging station placing and sizing. Applied Energy, 125, 289-299. http://dx.doi.org/10.1016/j.apenergy.2014.03.077.

Sailors for the Sea. (2019). Carbon footprint. Retrieved in 2019, June 5, from http://www.sailorsforthesea.org/programs/green-boatingguide/carbon-footprint

Timilsina, G. R., \& Shrestha, A. (2009). Factors affecting transport sector CO2 emissions growth in Latin American and Caribbean countries: an LMDI decomposition analysis. International Journal of Energy Research, 33(4), 396-414. http://dx.doi.org/10.1002/er.1486.

Union of Concerned Scientists. (2018). Electric vehicle battery: materials, cost, lifespan. Retrieved in 2019, May 10, from https://www. ucsusa.org/clean-vehicles/electric-vehicles/electric-cars-battery-life-materials-cost

Villa, D., \& Montoya, A. (2018). A taxonomy of energy consumption models for electric vehicles. In MOVICI-MOYCOT 2018: Joint Conference for Urban Mobility in the Smarty City. Medellin: IET. http://dx.doi.org/10.1049/ic.2018.0016.

Vutetakis, D., \& Wu, H. (1992). The effect of charge rate and depth of discharge on the cycle life of sealed lead-acid aircraft batteries. In Proceedings of the IEEE 35th International Power Sources Symposium (pp. 103-105). New York: IEEE. http://dx.doi.org/10.1109/ IPSS.1992.282019.

Yi, Z., \& Shirk, M. (2018). Data-driven optimal charging decision making for connected and automated electric vehicles: a personal usage scenario. Transportation Research Part C, Emerging Technologies, 86, 37-58. http://dx.doi.org/10.1016/j.trc.2017.10.014. 